\title{
Autonomous and Mobile Prototype of Curvature Sensor with Remote Reliable Communication of Spectral Curvature
}

\section{Francisco Bulnes', Isaías Martínez², Rocio Cayetano3 , Omar Zamudio², Cuauhtémoc Gutierrez ${ }^{2}$, Isaí Martínez ${ }^{4}$}

\author{
${ }^{1}$ Research Department in Mathematics and Engineering, TESCHA, Federal HighwayMexico-Cuautla Tlapala "La Candelaria", \\ Chalco, Mexico \\ ${ }^{2}$ Electronic Engineering Division, TESCHA, Federal Highway Mexico-Cuautla Tlapala "La Cande-laria”, Chalco, Mexico \\ ${ }^{3}$ Informatics Engineering Division, TESCHA, Federal Highway Mexico-Cuautla Tlapala "La Can-delar-ia", Chalco, Mexico \\ ${ }^{4}$ Electronics and Electrical Engineering Department, Technological Institute of Querétaro, Santiago De Queretaro, Mexico \\ Email: francisco.bulnes@tesch.edu.mx
}

How to cite this paper: Bulnes, F., Martínez, I., Cayetano, R., Zamudio, O., Gutierrez, C. and Martínez, I. (2016) Autonomous and Mobile Prototype of Curvature Sensor with Remote Reliable Communication of Spectral Curvature. Journal of Sensor Technology, 6, 159-179.

http://dx.doi.org/10.4236/jst.2016.64012

Received: August 17, 2016

Accepted: December 27, 2016

Published: December 30, 2016

Copyright $\odot 2016$ by authors and Scientific Research Publishing Inc. This work is licensed under the Creative Commons Attribution International License (CC BY 4.0).

http://creativecommons.org/licenses/by/4.0/

\begin{abstract}
Through the creation and construction of a curvature sensor of accelerometer type, using the spectral curvature concept or curvature energy that measures curvature in Volts $/ \mathrm{m}^{3}$, an autonomous and mobile censorship of curvature sensing with reliable data transmission/reception in real time and remote position is designed and constructed considering the spectra of curvature of the measured curvature energy during the advance of the prototype as the normed measure by $\left\|\nabla_{X} Y\right\| \leq C^{\beta}$, with $\beta$, a constant rationalized parameter according with the required advance of the mobile device in the control scale of their velocity. Likewise, the sensed curvature data are digitalized through wireless interconnectivity using a HC-05 Module with a programmable device that includes logic blocks whose interconnection and functionality can be configured according to the sensor measure in situs. Also an application is planted to the obtaining of an energy plus due to the curvature that could be used in the displacement of a vehicle.
\end{abstract}

\section{Keywords}

Censorship, Curvature Energy, Curvature Sensing, Curvature Sensor, Curvature Spectra, Mobile Curvature Sensor, Reliable Data

Transmission/Reception 


\section{Introduction}

Along several researches and works on integral geometry theory to field observables as curvature and torsion of the space, we have constructed an adequate energy integrals theory obtaining important results to international level of curvature given as smooth embedding of a differential sub-manifold given in co-cycles [1] [2] [3] [4] [5] and their application through electronics and science of specialized sensors obtaining the concept of curvature energy, and their application in a range of energy given by the interval [1] [3] [4] $[V]^{2} \int_{C} h k^{2} \mathrm{~d} s \geq\left(\int_{C} h^{2}-k\right)^{2} \mathrm{~d} s \geq \frac{1}{2} A V^{2} \int_{0}^{2 \pi} k(\theta) \mathrm{d} \theta$, where $V$, is the applied potential energy of curvature (in this case voltages) ${ }^{1} ; A$, is the area of the surface and $h$, their mean curvature and the last integral corresponds to the curvature energy employed to measure the roundness in their displacement along a principal direction. Applying certain cycles as Gaussian pulses which are measured invariant of curvature, we obtain the corresponding energy co-cycles that are curvature information "in situs" of the curvature sensor designed to first prototype of this type.

In the development of the different prototypes of curvature sensors and their study [1], the creation arises in the mobile technologies' context of a dynamical system of curvature sensing that has the optimal conditions in the transmission/reception process, likewise as their reliability in the communication process of curvature data through the energy spectra of curvature obtained by the curvature sensor in movement pre-establishing the real time and remote position conditions of the curvature sensor in the place where this is, due to their displacement.

The communication problem that arises is in the wireless interconnectivity which must be synchronized with the displacement of the mobile curvature sensor, furthermore considering the elimination of possible vibrations that can be transmitted to the mobile curvature sensor due to the proper ground, and that could be included as noise in the curvature data that are transmitted to their exploring. The autonomy and independence must be two characteristics necessary to the remote development of the prototype considering their stability, mechanical robustness which will be characterized by their traction as continuous function of displacement and of the direction change of the mobile curvature sensor. Our curvature sensor is obtaining a Gaussian curvature through electrical pulses (electrically gauged [2]) that arise of the energy curvature accumulated in the proper space or surface [1] [3] according to field theory.

The wireless interconnectivity must obey a relation between the transmission/reception of spectral curvature which must be a continuous transformation in a $\omega$-space [3] which obeys an intensive property of the space which is curvature in this case [4]. The remote sensing problems can be solved with the regularity in the inverse problems ${ }^{2}$ of $\kappa\left(\theta_{1}, \theta_{2}\right)$, (an integral transforms problem) which is a consequence of a good wireless

\footnotetext{
${ }^{1}$ This potential energy of curvature could be represented in an electronic application as voltage. The integral expression is a censorship of curvature condition which is a Hilbert inequality as given in (1).

${ }^{2}$ Problem in mathematics that defines an initial state of a process has arbitrary states after. In our problem, the initial state that must to determine is the field state to measure curvature considering the model of curvature energy in Gaussian pulses.
} 
interconnectivity designed from a microcontroller and the sensor in the remote system (see the Figure 1). Then a remotely sensed curvature signal at wavelength $\lambda$, can be written as:

$$
K=\int_{-\infty}^{\infty} \int_{-\infty}^{\infty} \mathrm{e}^{i\left(\theta_{1} v_{1} / 1_{1}+\theta_{2} v_{2} / \lambda_{2}\right)} \kappa\left(\theta_{1}, \theta_{2}\right) \mathrm{d} \theta_{1} \mathrm{~d} \theta_{2},
$$

where $v$, is called the phase speed (magnitude of the phase velocity) of the wave and $\boldsymbol{\theta}=\left(\theta_{1}, \theta_{2}\right)$, is the wave's frequency. One difficulty related with the solving of the integral equation related with the integral transform $\kappa\left(\theta_{1}, \theta_{2}\right)$, is when is presented instability (vibrations or noise), which arises as errors of the data programming or of their interfaces when the physical data is processed in spectral data. Then can arise one error which carry outputs from the $\omega$-space as $K+\varepsilon$, being $\varepsilon$, the transmission error for any physical question that affects the transmission. Then we need a condition over speed that is involved in the kernel of the integral transform (1) used in the corresponding inverse problem. We need search to do $\varepsilon \rightarrow 0$, when certain $\alpha \rightarrow 1$, in a structure equation that comes from $\operatorname{Hom}_{K}(M, \Sigma)=\mathbf{1}_{M}{ }^{3}$ [2] [5] (see the Figure 2).

The independence traction in the four tires to the mobile system of the curvature sensor reduces the vibrations offsetting the mass forces that are produced by the vibrations and that these can be transmitted to the sensor, that is to say, create an anisotropic elastically system, where the deforming and stress mechanisms in the inner as well as

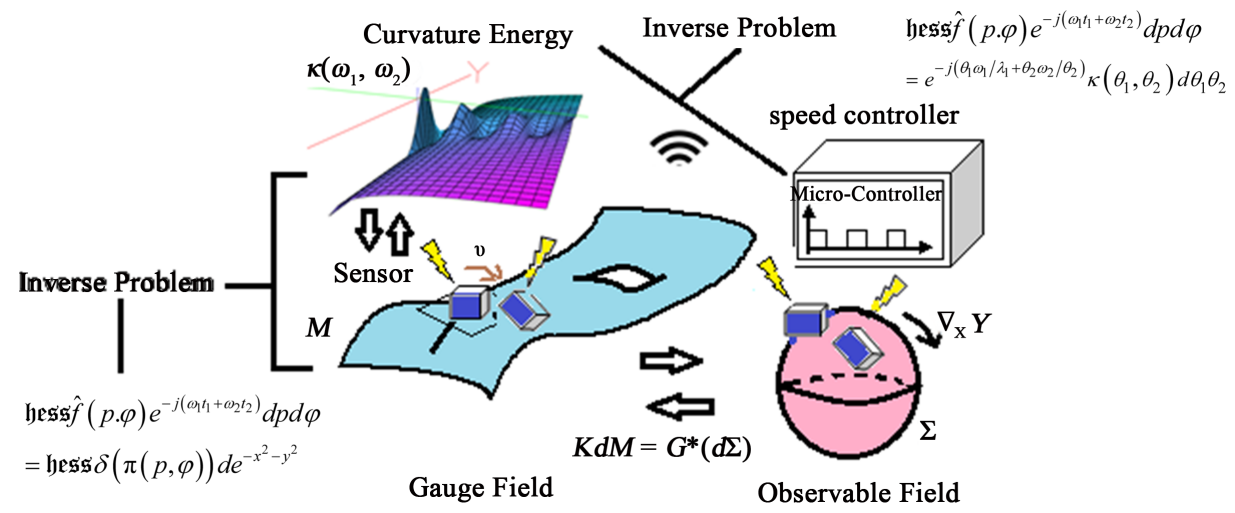

Figure 1. Gauge field is the measured by the accelerometer. The observable field is the reference systems field to measure curvature through Gaussian curvature. In the pictorial diagram are showed the inverse problems involves with the spherical mapping as fundamental patron to measure curvature in 2-surfaces [2] [5]. Observe as is necessary to design a control with constant pulses to transmission encoding of real data in the advance of our curvature sensor over $M$. The relation between sphere and micro-controller must be regularized by a relation of the derivative $\nabla_{X} Y$, and speed field which can be rectified by constant pulses in a speed control. This hypothesis is accord with the homogeneous space whose classes are in the sphere $\Sigma$. The speed rate over sphere is constant.

${ }^{3}$ Spherical mapping of the 2-dimensional surface (gauge field) and 2-dimensional sphere (reference systems field). Remember that [2] $\operatorname{Hom}_{K}(M, \Sigma)$, describes completely the turns that gives a normal and unitary vector in a neighborhood of a point $p \in M$. The gauge field is given by the electromagnetic field used to measure curvature [2]. 

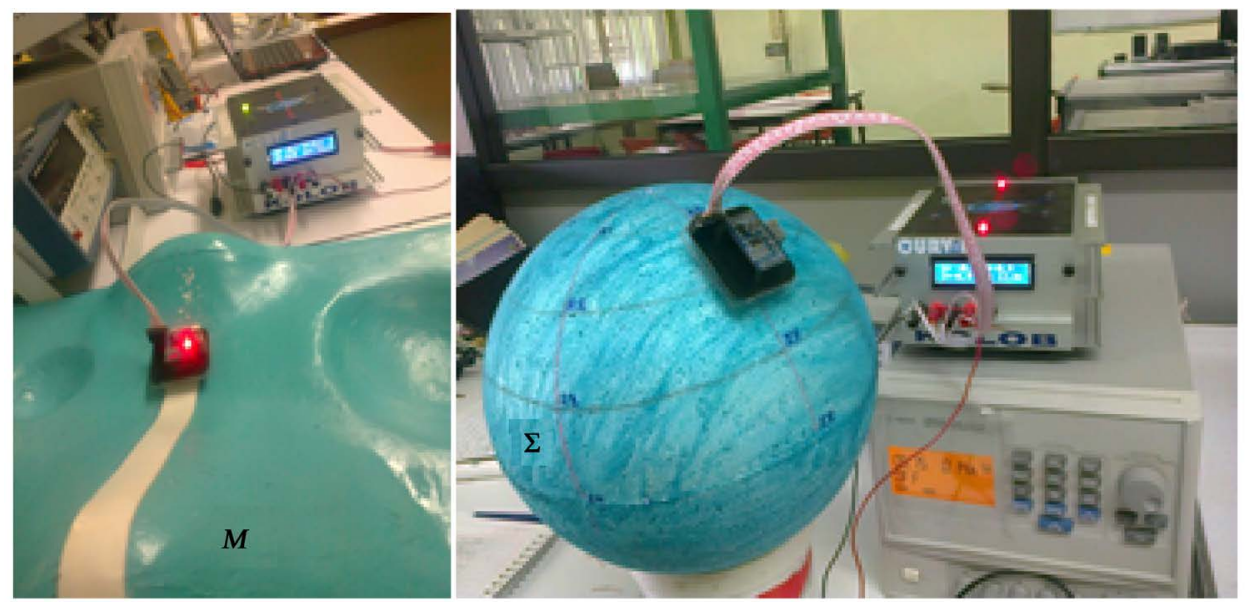

Figure 2. Experiments realized in electronics laboratory to verify $\operatorname{Hom}_{K}(M, \Sigma)=\mathbf{1}_{M}$. In these experiments is verified that the reference systems field has electrical field characterized to the sensorship [2] [5].

outside the mobile system not have inherence in the field observable measure. The spectral curvature is not deformed or noise contaminated. For it, we can consider an equilibrium equations system that will be constructed starting from the structural equations of curvature through of the field of observation ${ }^{4}$ (field of reference systems).

Then we will focus our study to the velocity advance of our mobile prototype which will be conditioned to the data Wi-Fi-transmission or data Bluetooth transmission velocity of our curvature sensor. As we want a constant data transmission then the velocity of advanced will be constant too. One our problem will be design a control of velocity in the mobile prototype such that the velocity field created by the reference systems field, obeys the fundamental equation

$$
v=\alpha\left\|\nabla_{X} Y\right\|,
$$

As a second problem that we want to solve and improve is on the energy optimization, where at the same time be measured the curvature could be used their curvature energy for optimization and performance of own sensor system, and have the better functioning of this.

${ }^{4}$ As field of reference systems must be understood al field $X=\left(E_{1}, E_{2}, E_{3}\right) \in \mathcal{X}\left(\mathbb{E}^{3}\right)$, such that $\forall E_{1}, E_{2}, E_{3} \in \mathrm{E}^{3}$, is had that $E_{i} \cdot E_{j}=\delta_{i j},(1 \leq i, j \leq 3)$, where $\delta_{i j}$, is the Kronecker's delta. Then the average with that the gauge field used in the detection and measure of curvature varies respect of the observation field is given as the covariant derivative of the vector fields of the reference systems in terms of the self-vector fields: $\nabla_{v} E_{i}=\sum_{j} c_{i j} E_{j}(p)$, where $p \in M, v \in T_{p} M$, and $c_{i j}=\nabla_{v} E_{i} E_{j}(p)$, with $1 \leq i, j \leq 3$. Here $M$, is the space, object of the exploration and measurement of their curvature. One best form of express the coefficients $c_{i j}$, (that depend of the tangent vector $v$ ) is through of the 1 -forms or connection forms $\omega_{i j}$, which will denote certain functions over arbitrary tangent vectors and with real values. Then the average of the field respect other field (in this case the field of reference systems) comes given by $\nabla_{v} E_{i}(p), \forall p \in M$. To our electronic sensor this can be re-interpreted as the electric field acting through voltage $/ \mathrm{m}^{3}=$ curvature units, [2] varying respect of the position average field registered in our accelerometer and whose energy curvature is the curvature spectra given by energy determined for their connections $\omega_{i j}$, and calculated for the integrals $\kappa\left(\theta_{1}, \theta_{2}\right)$, as has been established in [3]. 


\section{Regularity and Reliability of the Inverse Problem to Remote and Real Time Curvature Sensing}

The regularity problem must to be normed by the transmission velocity problem, which from a point of view of functional analysis has that see with order of a constant that must bounded the norm of the derivative to certain order.

In a transmission problem of the mobile devise the velocity of feeding of reference system field data must be normed by the velocity of the displacement of the device such that this last velocity is constant.

Lemma (regularity) 2.1. $\left\|\nabla_{X} Y\right\| \leq C^{\beta}, \beta \in Q$.

The velocity of response of the curvature sensor must be minor or equal to a constant $C^{\beta}>0, \forall \beta \in Q,{ }^{5}$ (that is to say, a multiple factor of displacement).

Proof. We consider the spherical mapping over $\Sigma$, (the sphere illustrated in the Figure 2), that is to say, $\operatorname{Hom}_{K}(M, \Sigma)=\mathbf{1}_{M}$, and we define a shape operator to this spherical mapping as [6]:

$$
S: T_{p}(M) \rightarrow T_{p}(\Sigma),
$$

with rule of correspondence $S(u)=-\nabla_{u} U$, where $U$, is a normal unitary field and $u$, is a unitary tangent vector to $M$, our 2-dimensional surface in $E^{3}$. We select a shape operator (which we can do it due to spherical mapping) such that

$$
\left\|\nabla_{u} U\right\|\|u\| \leq v
$$

It's necessary to prove that to the curvature signals detected by our curvature sensor their data transmission velocity must be bounded by a constant along of the space walked made by the sensor in movement, that is to say, their displacement velocity. First we must to prove that the process of transmission is given as continuous transformation of curvature energy data to the corresponding inverse problem. After, we use this to find the bound of velocity in this curvature context or geometrical context.

We consider the curvature integral operator as the spectral curvature in the direction $u$, over tangent straight line in tangent plane $T_{p}(M)$, then the normal component of the energy to the curvature (which is the energy that could be required to that the space $M$, can be curved or bended in $\mathrm{E}^{3}$ ), is the curvature energy given by the integral operator ${ }^{6}$ :

$$
k(u, \xi)=\int_{M^{2}} S(u) u \delta(\xi(s)) \mathrm{d} s,
$$

But we need verify the transmission process mobile sensor system-micro-controller,

\footnotetext{
${ }^{5}$ The succession of velocities in the sensor data transmission obeys to an asymptotic decreasing of velocity of the data emission system which will be found in the reception system and likewise successively in $p s$. The design must be such that velocities of the sensor data transmission as well as their mobile system must to be equaled at the infinity due our theory.

${ }^{6} \mathrm{We}$ have the two integrals of normal curvature to each component of unitary tangent vector:

$\int_{M^{2}} k_{1} u_{1}^{2} \delta(\xi(s)) \mathrm{d} s$, and $\int_{M^{2}} k_{2} u_{2}^{2} \delta(\xi(s)) \mathrm{d} s$, where is considered the shape operator as the matrix

$\left(\begin{array}{cc}k_{1} \cos \theta & 0 \\ 0 & k_{2} \sin \theta\end{array}\right)$, and the unitary tangent vector as $u=e_{1} \cos \theta+e_{2} \sin \theta$.
} 
that is to say, the convolution $k(u) * \kappa\left(t_{1}, t_{2}\right)$. Indeed, for one side to the convolution $\left\|k(u) * \kappa\left(\omega_{1}, \omega_{2}\right)\right\|_{2} \leq B_{2,1}\|k(u)\|_{1}\left\|\kappa\left(\omega_{1}, \omega_{2}\right)\right\|_{2}{ }^{7}$. Then considering (5) we have:

$$
\begin{aligned}
\|k(u, \xi)\| & =\left\|\int_{M^{2}} S(u) u \delta(\xi(s)) \mathrm{d} s\right\| \leq \int_{M^{2}}\left\|\nabla_{u} U\right\|\|u\| \delta(\xi(s)) \mathrm{d} s \\
& \leq\|S(u)\|\|u\| \int_{M^{2}} \delta(x(s)) \mathrm{d} s \leq\|S(u)\|\|u\| \leq\|k(u)\|,
\end{aligned}
$$

But $\|u\|=1$, and the normal curvature as function of the unitary tangent vector satisfies that $\|k(u)\| \leq C^{\eta}\|u\|$, then finally $\left\|\nabla_{u} U\right\| \leq C^{\eta}, \eta \in \mathrm{Q}$. Due to that the shape operator can be constructed to any fields $X, Y$, that is to say, the spherical mapping is applied to all smooth 2-surface (differentiable surface), finally we have to some constant $\beta$, that include $\eta$, that $\left\|\nabla_{X} Y\right\| \leq C^{\beta}, \beta \in \mathbb{Q}$.

All processes are bounded to the transmission velocity, which takes as reference the velocity of the shape operator where this last defines implicitly the curvature.

The scaled gravitational energy used in the process of gauging of sensor mobile advance corresponds to the measured and gauged by the proper universal gravitation and considered by the spherizer operator $\mathcal{O}_{E}$, [1] [2] [5]. Then we can enounce:

Theorem (F. Bulnes) 2.1. (Scaled Gravitational Energy to Curvature Sensor advance $\left.{ }^{8}\right)$.

Let be the Einstein equations:

$$
R_{\mu \nu}-\frac{1}{2} R g_{\mu \nu}=G T_{\mu \nu},
$$

Let be the spherizer operator $\mathcal{O}_{E}$, defined on 2-dimensional surface $M$ [2] [5] to apply the sphere of radius $r$, (see the Figure 3$)^{9}$

$$
\mathcal{O}_{E}=d(\gamma) \operatorname{Hom}_{K}\left(M, S^{2}\right) \int_{M} \mathbf{e}(-1)^{n} \mathrm{~d} \Sigma(f(x))=8 \pi r^{2},
$$

Then the scaled gravitational energy of the curvature sensor advance certain length $L$, is

$$
\Theta_{E}=G\|k * \kappa\| L,
$$

Proof. The scaled gravitational constant is $G=\frac{8 \pi}{\mathbf{c}^{4}} G=2.071 \times 10^{-43} \mathrm{sec}^{2} \cdot \mathrm{meter}^{-1} \cdot \mathrm{kg}^{-1}$, which is the proportionality between space-time curvature and energy (see the Figure 3). In particular, to our 2-dimensional space, said proportionality is normed by the spherical mapping $\operatorname{Hom}_{K}(M, \Sigma)$, between 2-sphere applied to the 2-surface, having by [2] [5], an energy sphere equal to $8 \pi$ meter $^{2}$. Indeed, by the spherizer (6) we have:

${ }^{7}$ This is the energy given in Joules, where the constant $B_{2,1}$, will have units in meters. This corresponds to the advance of the device over surface $M$. Likewise, the dimensional analysis will be

$B_{2,1}\|k(u)\|_{1}\left\|\kappa\left(\omega_{1}, \omega_{2}\right)\right\|_{2}\left(=m_{2,1}\left(\frac{\mathrm{kgm}^{2}}{\mathrm{~s}^{2}}\right)_{2}\left(\frac{1}{\mathrm{~m}}\right)_{1}\right)$.

${ }^{8}$ It's the gravitational energy to an advanced accelerometer design. This could to carry us to measure field observable more fine as quantum gravity, in a future. In this case, the gauge field is a classical electric field characterized for voltages.

${ }^{9} d(\gamma)=2$. 


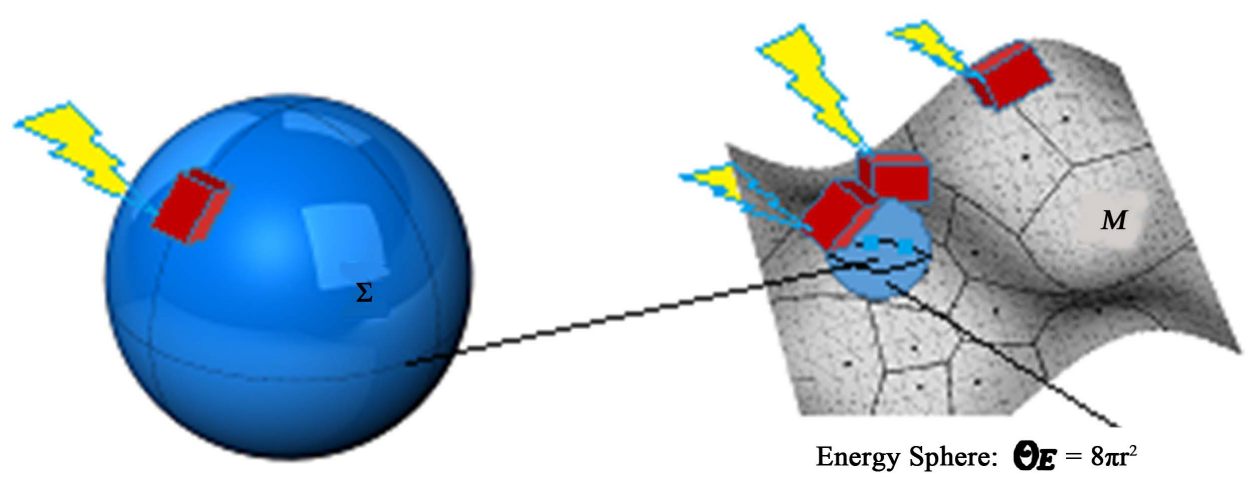

Figure 3. Scaled gravitational energy to curvature sensor.

$$
\mathcal{O}_{E}\left(=\frac{\mathrm{sec}^{2}}{\mathrm{~kg} \cdot \text { meter }} \times \frac{\mathrm{kg} \cdot \text { meter }^{2}}{\mathrm{sec}^{2}} \times \text { meter }\right)=8 \pi r \times L,
$$

Due to the symmetry of sphere, $L=r$, that is to say, the advance could be realized as if the sphere shot, doing with maximum length equal to radius (see the Figure 3) then is had (7).

\section{Design of the Wireless Interconnectivity Module and Transmission Spectra}

We consider the curvature signals (curvature energy) in real time $\kappa\left(t_{1}, t_{2}\right)$, whose transmission will be bounded $\leq C^{\beta}$, by a velocity control to the mobile device that will transport the sensor, doing it through a signal of constant rectangular pulses with their instantaneous sampling to an efficient transmission process in a mobile curvature sensor $^{10}$. We consider a curvature energy obtained in the spectra of energy constructed through Gaussian pulses [1] [3] (see the Figure 4).

Then the optimal signal in the transmission process will be the PWM resultant signal of plane ridge obtained in the sampling of the curvature energy signal (see the Figure 5 and Figure 6). In this process we have an inverse problem, where to the recovering of the real time curvature signal $\kappa\left(t_{1}, t_{2}\right)$, from their PWM signal $\kappa_{s}\left(t_{1}, t_{2}\right)$, (demodulation of the PWM signal) comes given by the process or product detector device:

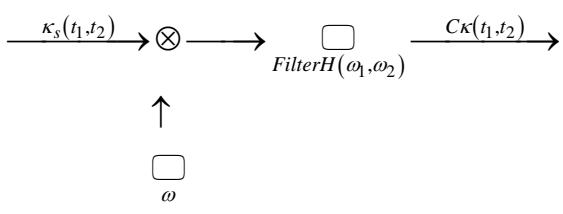

Due to the equation $\left\|\nabla_{X} Y\right\|=C^{\beta}$, is established the necessity of a constant velocity. For it, is necessary develop an electronic system of constant velocity control of the mobile automata; this constant velocity is necessary to that the development in straight

${ }^{10}$ The constant rectangular pulses have been chosen to control of vehicle velocity due to that in electronics tend to an impulse function when their length tends to zero, that is to say, the limit $\underset{\tau \rightarrow 0}{\operatorname{rect}}(t / \tau)=\delta(t)$. Likewise to a period we can conserve the electrical force of curvature signal using an impulse train derived of the sampling by rectangular pulses. The power of signal is conserved and transmitted in an interval in $p s$. 


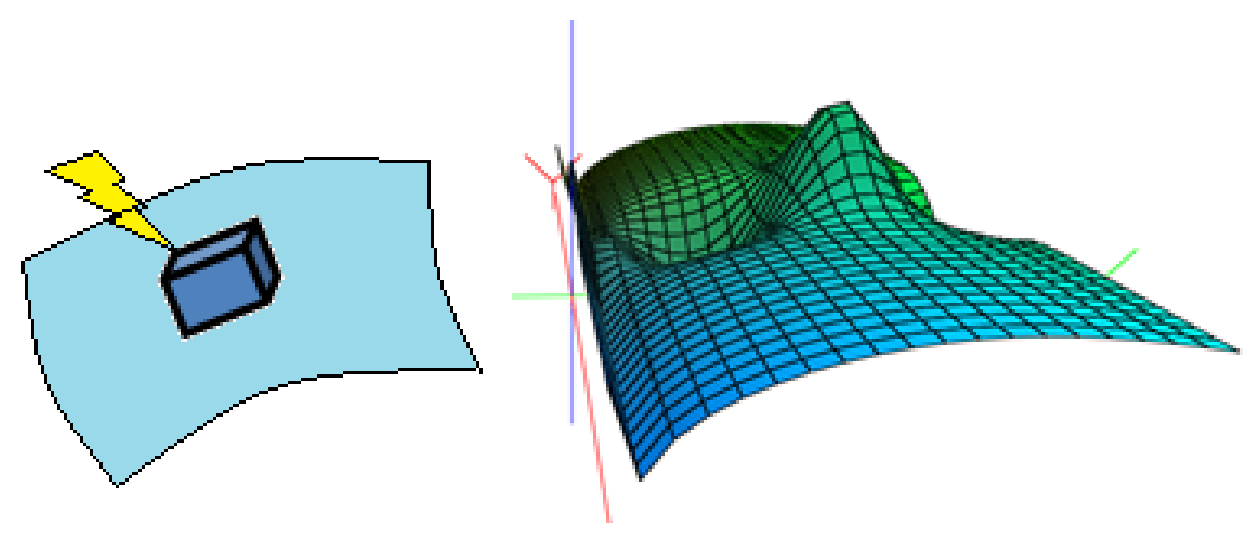

Figure 4. Curvature energy $\kappa\left(t_{1}, t_{2}\right)$.

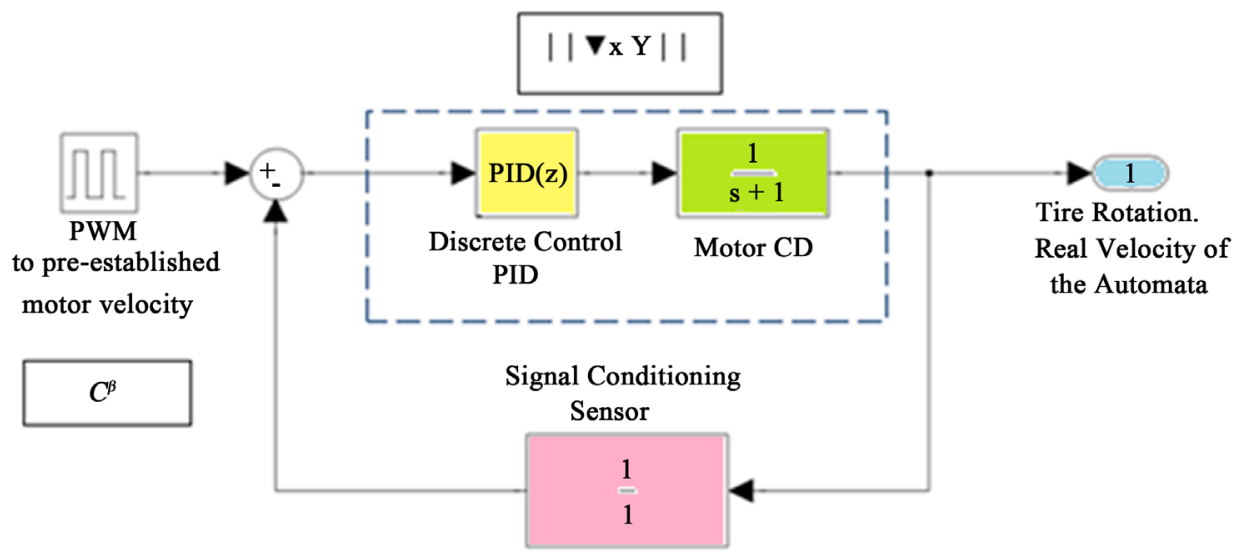

Figure 5. Closed-Loop system of discrete values, where is implemented a micro-controller to control the velocity. For it, is required a conditioning sensor of digital signal and to the power to the CD motor step a modulation of discrete pulse width (PWM). The relation of linear displacement, electric field variation in the accelerometer gives expressions such as $x=\pi D$.
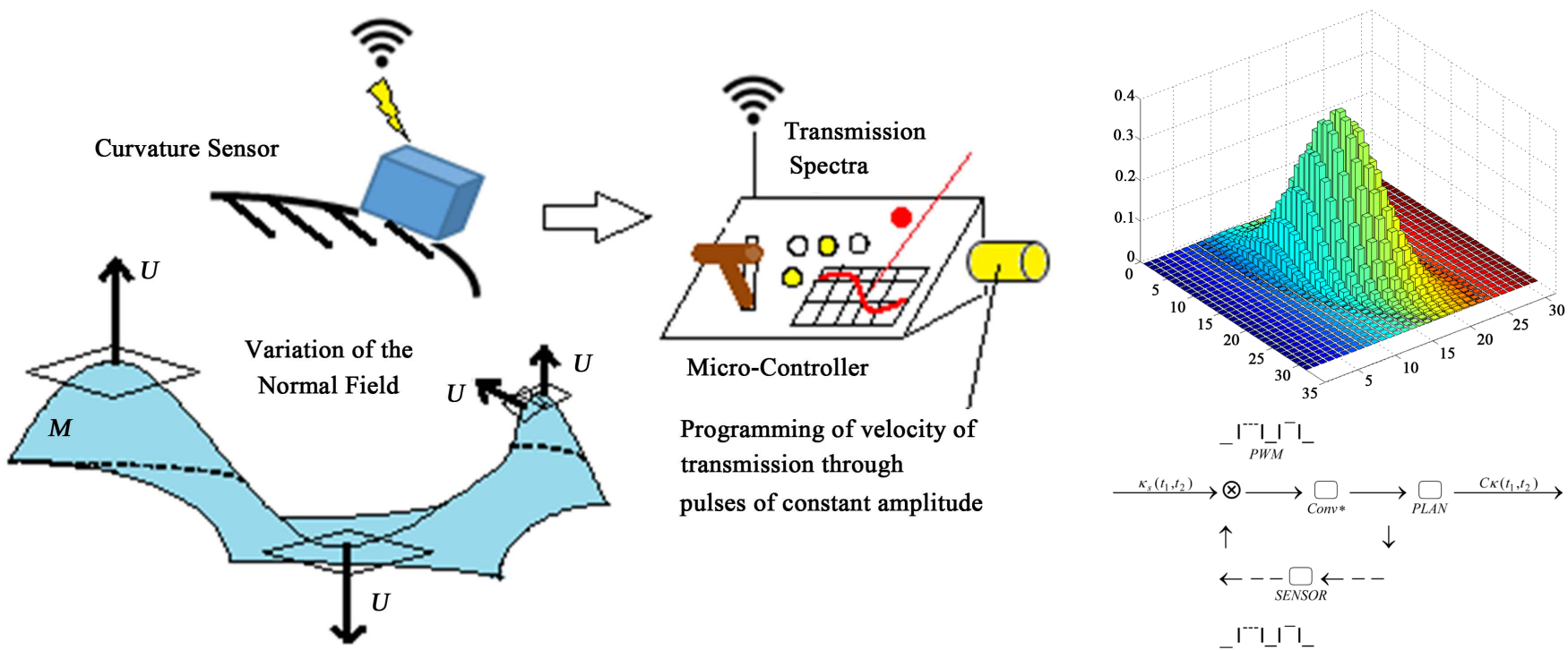

-

Figure 6. Velocity control of the mobile curvature device. Sampling spectral curvature. 
line exist slopes to upwards and downwards ensuring that the data transmission of the curvature sensor device to the wireless receptor device that is reliable.

Now to the control process of velocity to the vehicle of our mobile curvature sensor we have the diagram (Figure 5).

Likewise, if $\kappa\left(t_{1}, t_{2}\right)$, is the analogic curvature signal of band-limited to $B=10$ Hertz, their PWM sampled signal is given for:

$$
\kappa_{s}\left(t_{1}, t_{2}\right)=\sum_{n}^{\infty} \sum_{k}^{\infty} \kappa\left(n T_{1}, k T_{2}\right) p\left(t_{1}-n T_{1}, t_{2}-k T_{2}\right),
$$

where $p\left(t_{1}, t_{2}\right)$, is the rectangular pulse of sampling

$$
p\left(t_{1}, t_{2}\right)=\operatorname{Rect}\left(\frac{t_{1}}{T_{1}}, \frac{t_{2}}{T_{2}}\right)=\left\{\begin{array}{cc}
1 & \left|t_{1}\right|<T_{1} / 2,\left|t_{2}\right|<T_{2} / 2 \\
0 & \text { other case }
\end{array},\right.
$$

where to simplify the compute of data, we can have square pulses in our sampling, considering the condition $T_{1}=T_{2}$, with sampling frequency $0 \leq T_{s}=1 / \omega_{s}$, and $\omega_{s} \geq 2 B$, (see the Figures 6-8).

Proposition 3.1. The curvature $\kappa\left(t_{1}, t_{2}\right)$, has the sampling curvature energy of energy spectra of rectangular pulse $e^{11}$ :

$$
\kappa_{s}\left(\omega_{1}, \omega_{2}\right)=\frac{1}{T^{2}} H\left(\omega_{1}, \omega_{2}\right) \sum_{n}^{\infty} \sum_{n}^{\infty} \kappa\left(\omega_{1}-n \omega_{s}, \omega_{2}-n \omega_{s}\right),
$$

where

$$
H\left(\omega_{1}, \omega_{2}\right)=\mathcal{F}\left\{p\left(t_{1}, t_{2}\right)\right\}=\operatorname{sinc}\left(\omega_{1}, \omega_{2}\right) .
$$

(see the Figures 6-9).

\section{Experimentation and Analysis.}

We establish the bordering conditions. The velocity of the mobile servosystem that carries the sensor device (accelerometer) is considered minor much that the transmission velocity

$$
\begin{aligned}
& { }^{11} \mathrm{~A} \text { general proof of the proposition 3. 1, is: Consider the convolution: } \\
& \kappa_{s}\left(t_{1}, t_{2}\right)=\sum_{n_{1}}^{\infty} \sum_{n_{2}}^{\infty} \kappa\left(n_{1} T_{1}, n_{2} T_{2}\right) p\left(t_{1}, t_{2}\right) * \delta\left(t_{1}-n_{1} T_{1}, t_{2}-n_{2} T_{2}\right) \\
& =p\left(t_{1}, t_{2}\right) \sum_{n_{1}}^{\infty} \sum_{n_{2}}^{\infty} \kappa\left(n_{1} T_{1}, n_{2} T_{2}\right) * \delta\left(t_{1}-n_{1} T_{1}, t_{2}-n_{2} T_{2}\right) \text {; where the spectra is: } \\
& =p\left(t_{1}, t_{2}\right) * \kappa\left(t_{1}, t_{2}\right) \sum_{n_{1}}^{\infty} \sum_{n_{2}}^{\infty} \delta\left(t_{1}-n_{1} T_{1}, t_{2}-n_{2} T_{2}\right), \\
& \kappa_{s}\left(\omega_{1}, \omega_{2}\right)=H\left(\omega_{1}, \omega_{2}\right)\left[\kappa\left(\omega_{1}, \omega_{2}\right) * \sum_{n}^{\infty} \sum_{n}^{\infty} \mathrm{e}^{-j 2 \pi\left(\frac{n_{1} T_{1} \omega_{1}+n_{2} T_{2} \omega_{2}}{n_{1} T_{1} \rho_{1} T_{2} \omega_{2}}\right)}\right] \text {, But the sum of exponential functions is equivalent } \\
& \text { to an expression of the Fourier series in the } \omega \text {-space: } \\
& \frac{1}{T_{1} T_{2}} \sum_{n_{1}}^{\infty} \sum_{n_{2}}^{\infty} \delta\left(\omega_{1}-n \omega_{s}, \omega_{2}-n \omega_{s}\right)=\frac{1}{T_{1} T_{2}} \sum_{n_{1}}^{\infty} \sum_{n_{2}}^{\infty} c_{n_{1}} c_{n_{2}} \mathrm{e}^{-j 2 \pi\left\{n_{1} T_{1} \omega_{1}+n_{2} T_{2} T_{2}\right\}} \text {, where the amplitudes in the } \omega \text {-space are: } \\
& c_{n}=\frac{1}{\omega_{1} \omega_{2}} \int_{-\omega_{1} / 2}^{\omega_{1} / 2} \int_{-\omega_{2} / 2}^{\omega_{2} / 2}\left[\sum_{n_{1}}^{\infty} \sum_{n_{2}}^{\infty} \delta\left(\omega_{1}-n_{1} \omega_{s}, \omega_{2}-n_{2} \omega_{s}\right)\right] \mathrm{e}^{-j 2 \pi\left\{n_{1} T_{1} \omega_{1}+n_{2} T_{2} \omega_{2}\right\}} \mathrm{d} \omega_{1} \mathrm{~d} \omega_{2} \text {, Then } \\
& \kappa_{s}\left(\omega_{1}, \omega_{2}\right)=\frac{1}{T_{1} T_{2}} H\left(\omega_{1}, \omega_{2}\right)\left[\sum_{n}^{\infty} \sum_{n}^{\infty} \kappa\left(\omega_{1}, \omega_{2}\right) * \delta\left(\omega_{1}-n \omega_{s}, \omega_{2}-n \omega_{s}\right)\right] \text {, which is the resulted required. }
\end{aligned}
$$




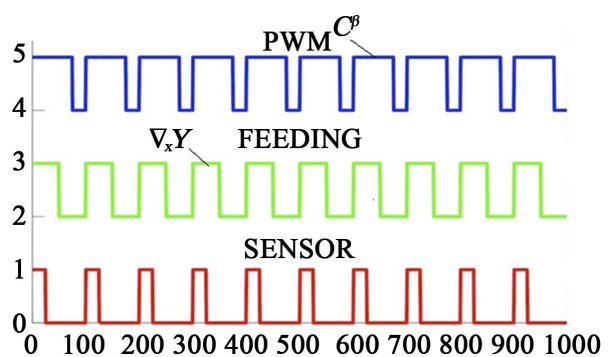

(a)

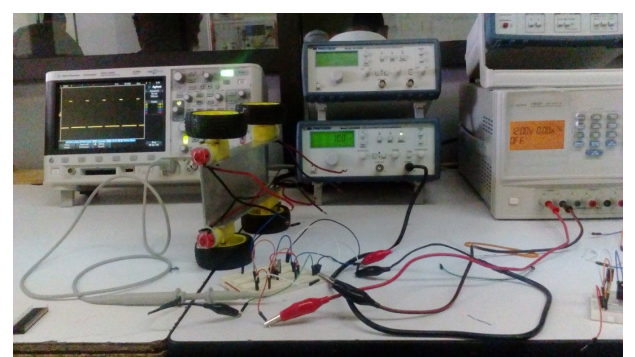

(b)

Figure 7. (a) Sample that act in the micro-controller; (b) Velocity control of the tires of mobile device.

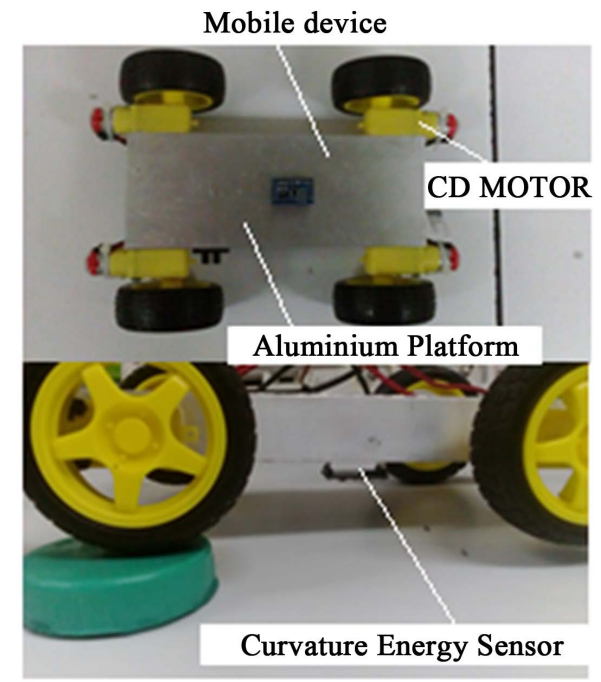

(a)

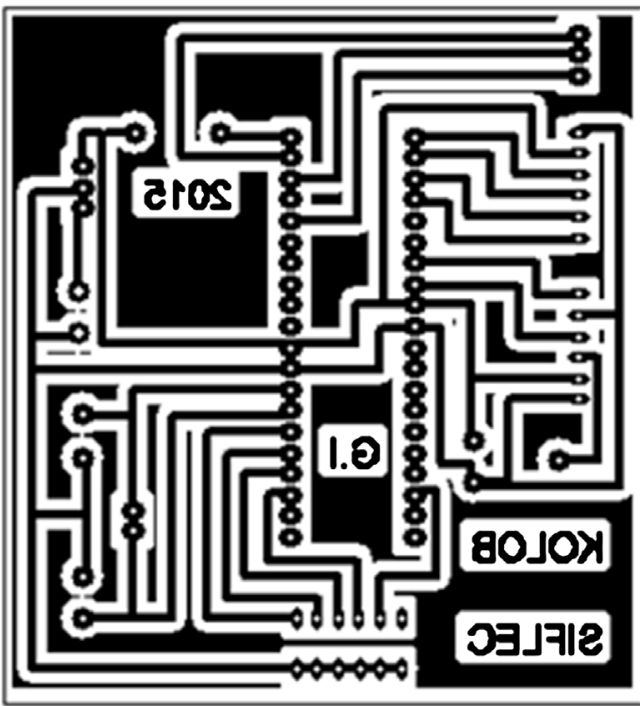

(b)

Figure 8. (a) Mobile curvature sensor prototype II (all the system); (b) Design in PCB Wizard to print circuited of the electronic board of the PIC16F877A Microcontroller and feeding 5V CD to the devices of curvature sensor. 


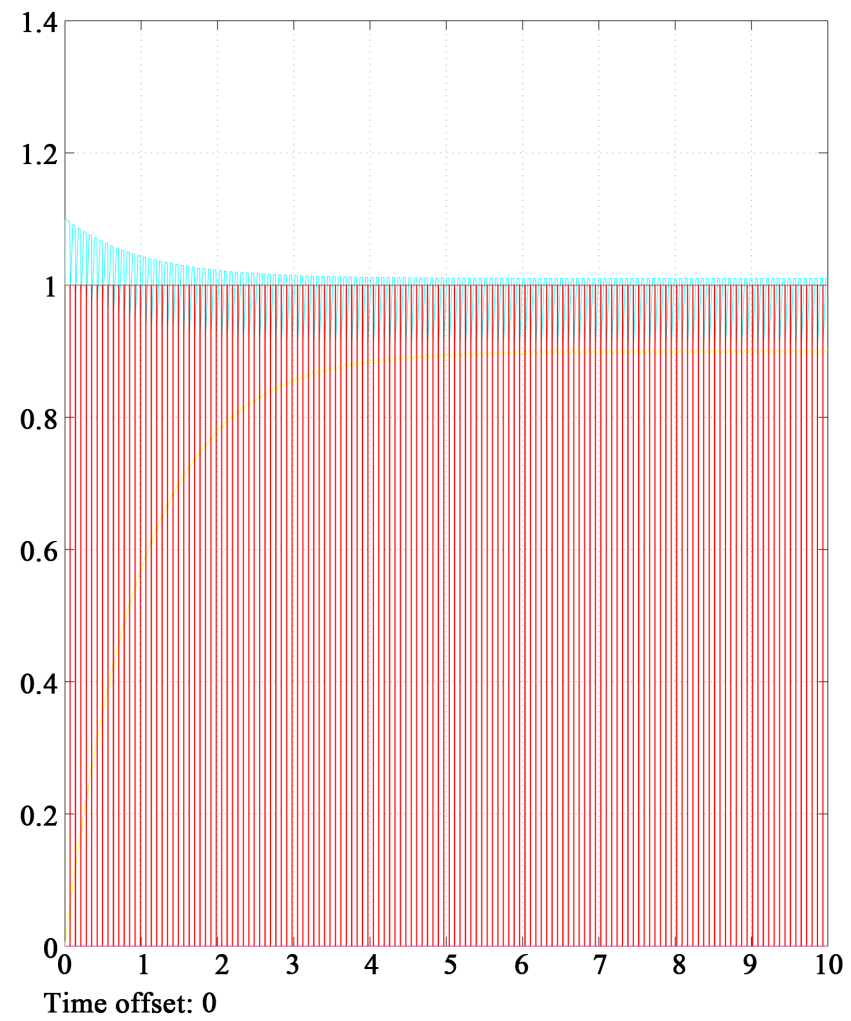

(a)

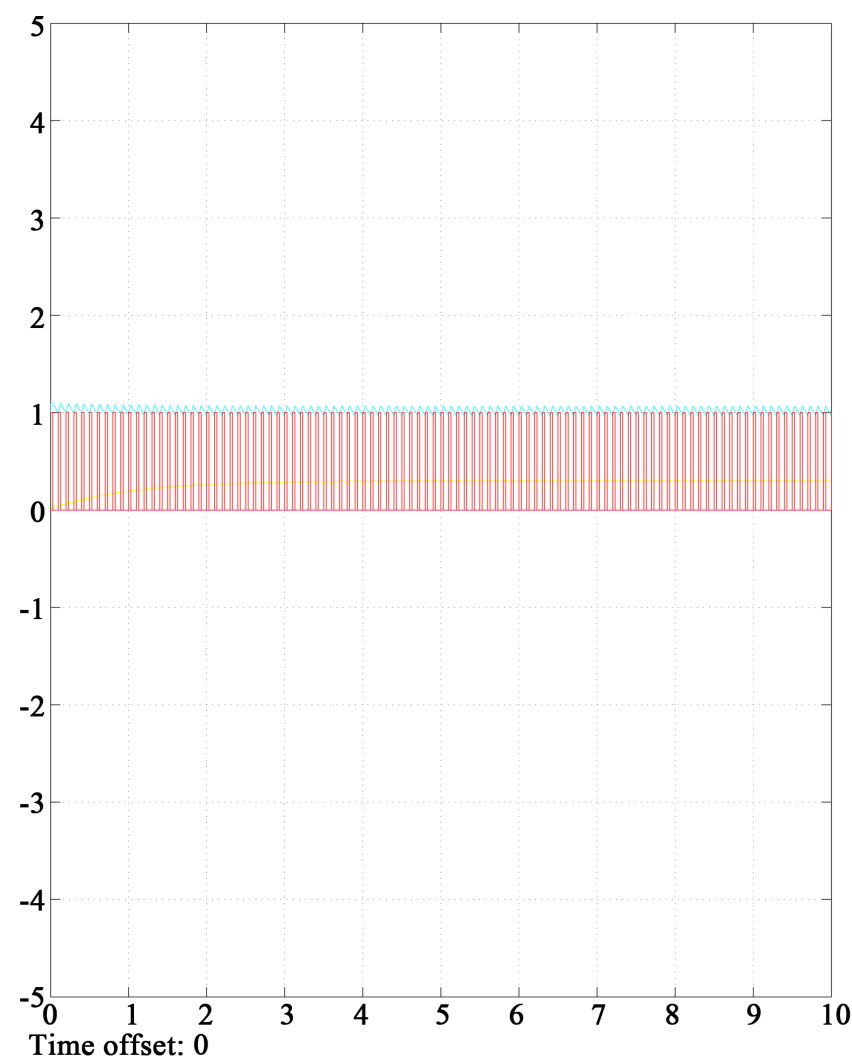

(b) 


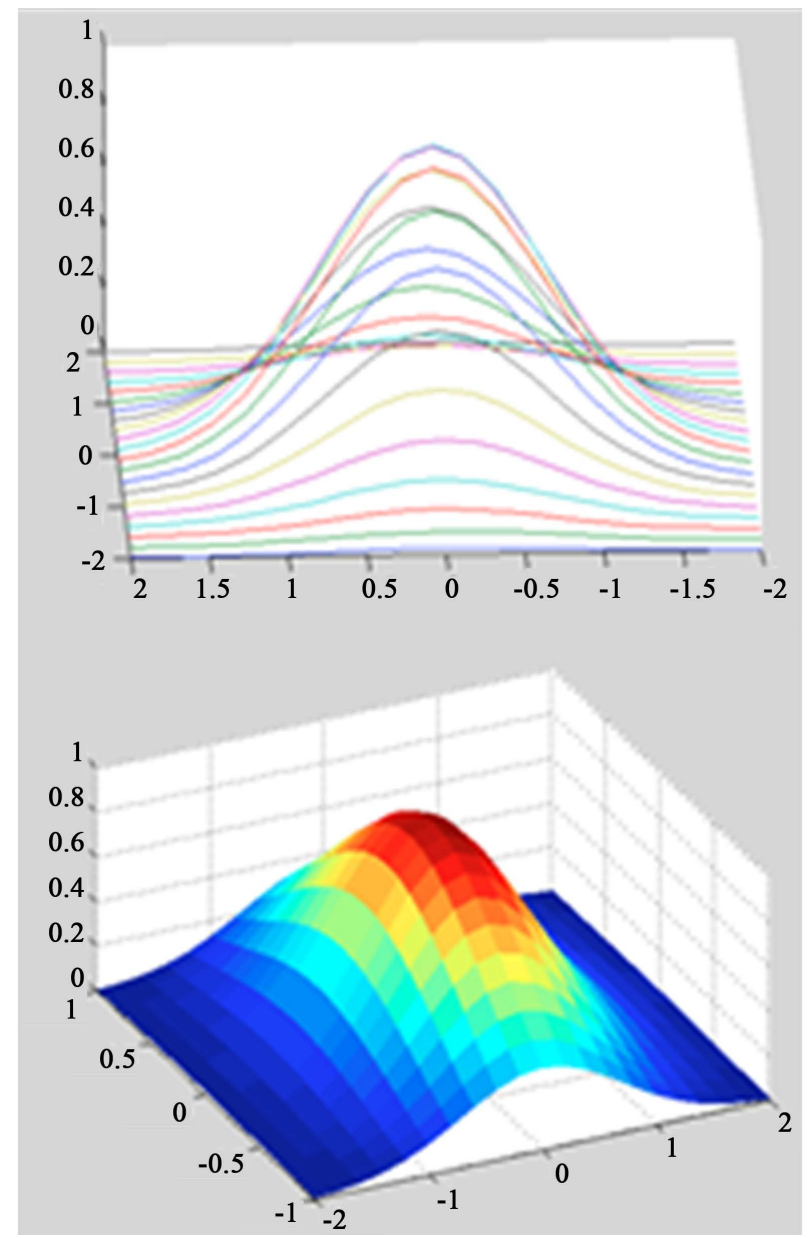

(c)

Figure 9. The graphs show in red color the PWM that feeds the reductor motors. The yellow color shows the torque in the motor arrow. The blue color shows the velocity in the pre-established straight line A). Variation of the electric field inside the sensor device due to their respective variable capacitance. This is translated in curvature measures.

$$
v(t) \ll c,
$$

where $v(t)$, is the constant linear velocity represented in addition for $C^{\beta}, c$, is the transmission velocity $\approx 3 \times 10^{8} \mathrm{~m} / \mathrm{s}$. For it is despised the error of data transmission in wireless modus, error that can give it due to the displacement of the mobile servo-system and the fix station of reception of data.

Likewise, to norm the velocity of the mobile servo-system we use the linear displacement that arise in the theoretical analysis of our energy integrals given in Equation (7) and applying this to a displacement in one direction. Then the velocity servo-system must to comply the following system equations:

$$
\begin{gathered}
v(t)=\frac{2 \pi r}{t}=\ddot{X}, \\
V_{i(t)}=R i(t)+L \frac{\mathrm{d} i}{\mathrm{~d} t},
\end{gathered}
$$




$$
\dot{\theta}=K e V_{i(t)}
$$

where $K e$, is a specific proportionality constant of the CD-motor between voltage and angular velocity. Likewise

$$
v(t)=K_{3} \dot{\theta}
$$

where $K_{3}$, is a proportionality constant between the linear velocity and angular velocity given $t$ is last, in $\mathrm{m} / \mathrm{rad}$. Then the relation of constant velocity that must to have the motor is proportional to the voltage through the constants $K e$, and $K_{3}$, that is to say, ${ }^{12}$

$$
v(t)=K_{3} K e V_{i(t)}
$$

where $K_{3} K e$, is given by $\mathrm{m} / \mathrm{sV}$, The voltage of feeding to the motors is a modulated discrete function in pulse width to maintain a constant velocity in the wheels, each one with independent traction but synchronic. To this analysis only is considered the relation of feeding voltage to the motors and their velocity (Figure 7(a) and Figure 7(b)). In this analysis is not considered the torque given in the arrow of the motors and their directly proportional relation to the electric current $i(t)$, point that will be necessary to take in account to an analysis of energy optimization of the autonomous mobile servo-system in other time. Then arise a question, how is modified the PWM (Figure 7(a)) to have a constant velocity in straight line to that the sensor can be stable?

We consider the following analysis of velocity and torque in motors. Let the equation system of motors:

$$
\begin{gathered}
V_{i}-V_{R}-V_{L}=0, \\
\tau-J \ddot{\theta}=0, \\
\dot{\theta}=K_{1} V(t), \\
\tau=K_{2} i(t),
\end{gathered}
$$

Substituting the voltage expression from (16) in (14), and considering the velocity transference function of each motor:

$$
\theta(s)=I(s)[R+L s] K_{1},
$$

to obtain the linear velocity with $0.06 \mathrm{~m}$ of radius of the wheels, (see Figure $7(\mathrm{~b})$ ),

We obtain (see blue line in the (Figure 7(a)):

$$
v(t)=(\pi \times 0.06)\left[I(s)(R+L s) K_{1}\right]
$$

Now the torque will be:

$$
\tau=K_{2} I(s)
$$

\footnotetext{
${ }^{12}$ In reality the Equation (13) comes from the condition established by the lemma 2.1. Then in a lemma sense can be written as $|v(t)| \leq K_{3} \mathrm{KeV}_{i(t)}$, depending of the expression to the Voltage.
} 
The dynamical analysis shows that the velocity is expressed in terms of the current through (19). The servo-system that is showed in the block diagram in Simulink is a $\mathrm{CD}$ motor of unique phase mechanically coupling to a rotational-traction system that finally as output variable is a velocity in constant straight line (see the Figure 8(b)).

The automata being in their travel through a slope in ascent requires major traction force thus their feeding of the motors requires in their corresponding width modulation of pulse, major time in high voltage state that implicitly is available as electrical current necessary that demands the proper servo-systems in ascent (see the Figure 9(a)).

For other way, the contrary case in the moment of decline is required the decreasing of the velocity because is reduced the pulse width in high voltage state, such that the motors to not receive constant voltage then their velocity decreases to compensate the velocity that the servo-system sums when for gravity tends to go down (see the Figure 9(b)).

The modulation of pulse width PWM, whether in raising or lowering has variation, and exists a relation of this signal with the inertia of the servo-system in their constant movement and thus also with the variation of the electric field inside the sensor device due to their respective variable capacitance according the position that this has, which is translated in curvature measure (see the Figure 9(c)).

In laboratory the before analysis is verified and proved (see the Figure 10). The signal obtained in the oscilloscope shows the discrete signal used to excite the system, to difference of the analysis made in the Figure 9(a) and Figure 9(b).

\section{Transmission Optimization}

Due to the covering of field energy the data transmission of curvature energy will be given in bluetooth transmission whose decoding module use the energy interval [1] [3] established in a beginning to data whose transmission velocity is most speed. We require a transmission secure system of data with a band-width adequate to the norm established to velocity of curvature data transmission. We elect the bluetooth HC-05 y and the FPGA transmission model whose manufactured components have TTL technology with TTLRS elements of input-output of device known as bipolar transistors (th. [7].

The Bluetooth HC-05 module, is the that offers a best relation of price and functioning characteristics since is a master-slave module, which want to say that furthermore of receive connections from a PC or tablet, also is able of generate connections to other Bluetooth devices. This permit us, for example to connect two Bluetooth modules and form a connection point to point to transmit data between two micro-controllers or devices, as could be the case in our mobile curvature sensor [8]. The transmission modus will be the asynchronous, since offer us the advantage of that the bits that constitute the code of a character of the sampling spectral curvature $\kappa_{s}\left(\omega_{1}, \omega_{2}\right)$, can be emitted with the help of supplementary impulses that permits to maintain in synchronism the two extremes of their binary $\operatorname{codes}^{13}$.

${ }^{13}$ This communication method is more efficient due to transmission velocity. It comes given because there is not nobody additional information type between the characters to be transmitted. 
DS0-X 2004A, MY53100150: Fri Sep 09 02:38:59 2016

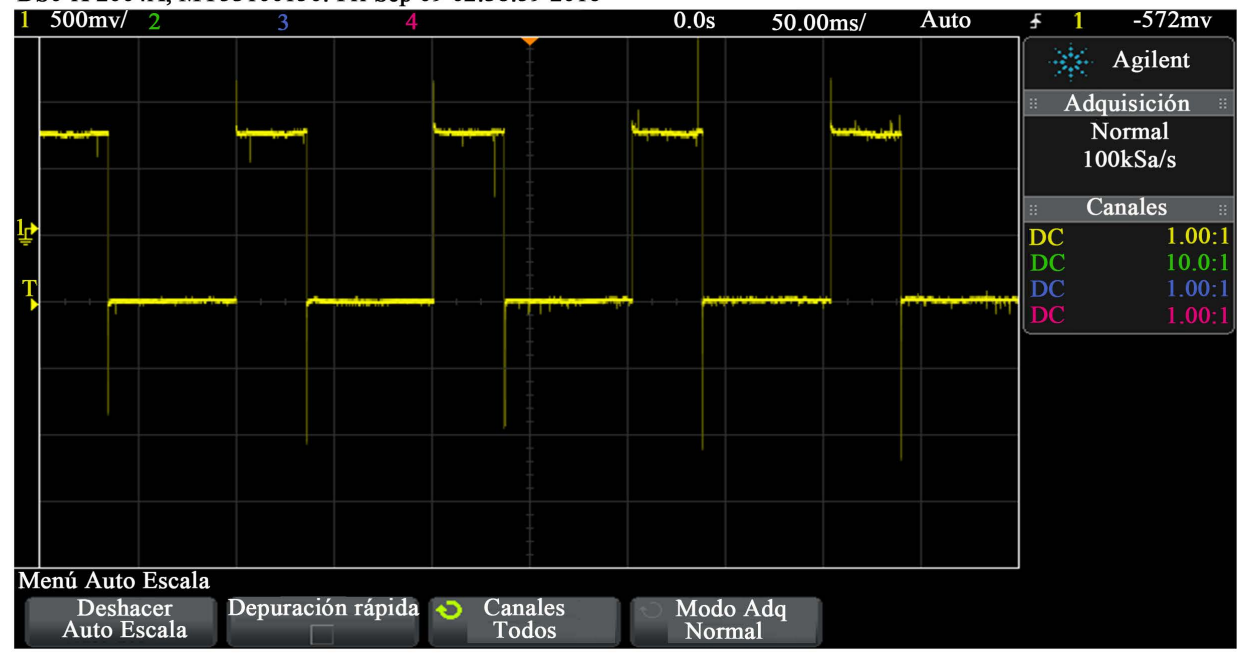

(a)

DS0-X 2004A, MY53100150: Fri Sep 09 02:41:27 2016

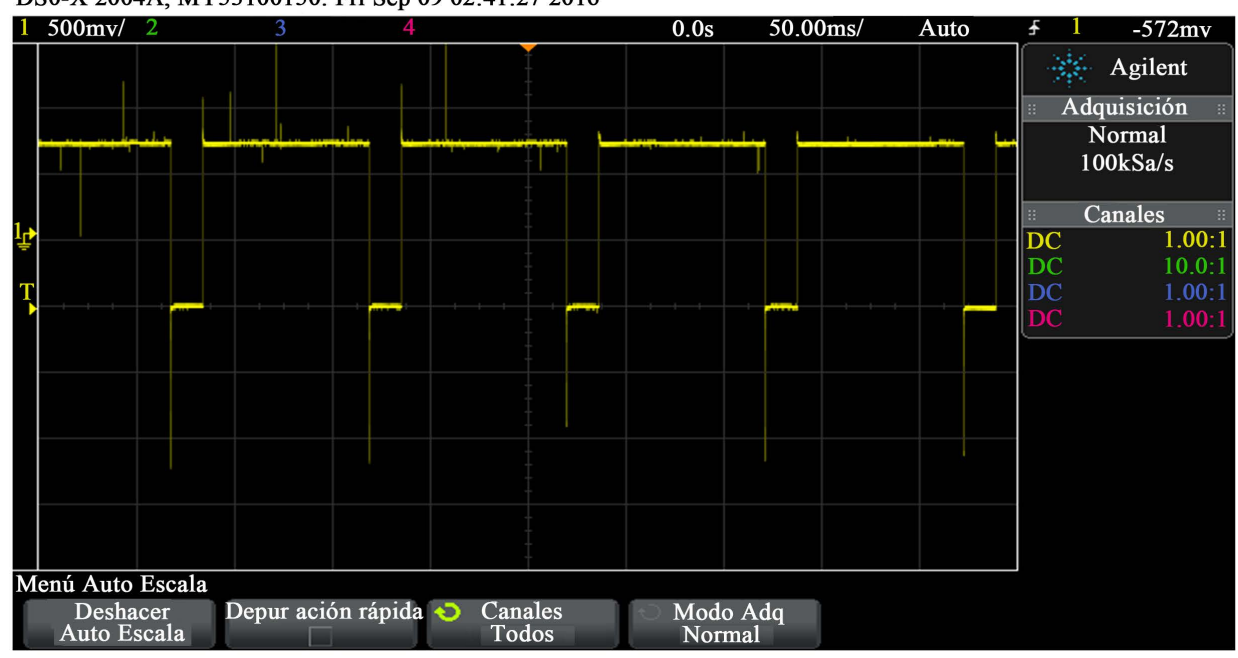

(b)

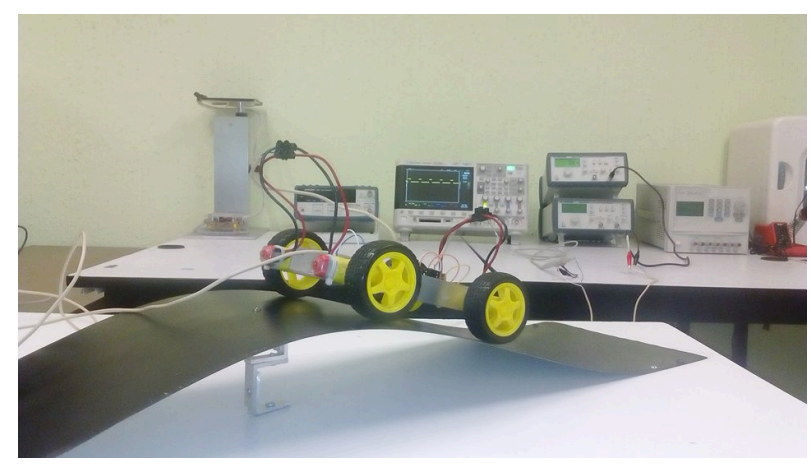

(c)

Figure 10. (a) The figure shows the measured signal with $30 \%$ of pulse width to $10 \mathrm{~Hz}$; (b) The figure shows the measured signal with $90 \%$ of pulse width to $10 \mathrm{~Hz}$; (c) Mobile curvature sensor device rising in one direction of constant movement governed by velocity control of order $\left\|\nabla_{X} Y\right\| \leq(\pi \times 0.06)\left[I(s)(R+L s) K_{1}\right]$. 
Communication via Bluetooth from a cell to the FPGA to test obtaining bits sent. Android application is made, the application is installed cell and connects to the HC-05. Given the connection a program structured VHDL is made to connect with the Bluetooth module and therefore the information as programmed in the FPGA is processed. The data output ports of the FPGA are sent. Physically they show each bit by LEDS [9] [10].

The serial data transmission allows the scope of the data is greater, since the form of higher performance parallel transmission system and transmission range is less demand. The RS-232 communication is made to review and store data processing on the computer, an interface defined chart in order to visualize the bits of information, which in turn are stored to be analyzed late [8].

The tests and rehearsals in laboratory show the binary encoding using the transmission optimization characterizes signed in the curve (Figure 11(b)) and executed by the Bluetooth module HC-05 (Figure 12). The additional equipment required for the purposes of digital wireless communications is:

1) It requires two HC-05 Bluetooth modules for transmission and reception of data from the PLC (see the Figure 12(a)).

2) FPGA is required for processing the data obtained. A USB-SERIAL wire is used for the connection the FPGA to computer (see the Figure 12(a) and Figure 12(c)).

3) A computer with a hyper-terminal program is required to achieve RS-232 connection of the FPGA and store the acquired data (see the Figure 12(c)).

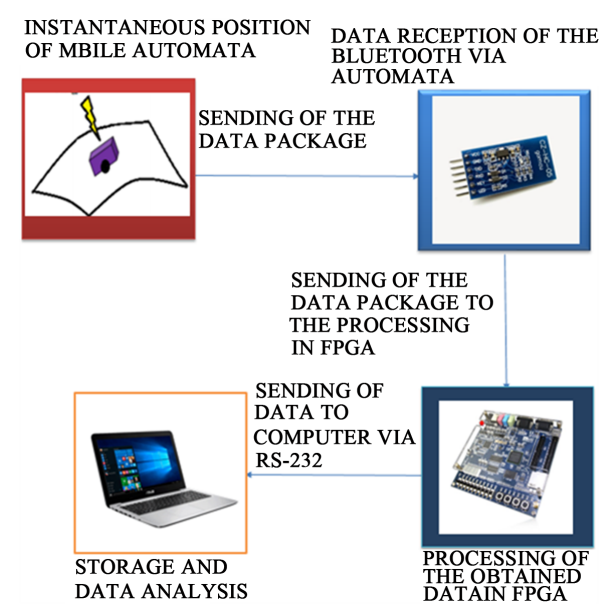

(a)

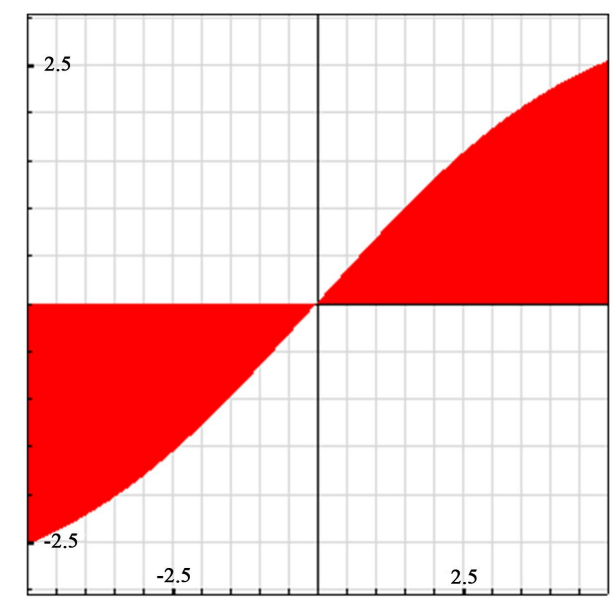

(b)

Figure 11. (a) A wireless transmission via Bluetooth is proposed. bluetooth module HC-05 is used because of its high efficiency in digital communications and receiver feature/transmitter will choose to use a Field Programmable Gates Array ( FPGA) due to its high speed data processing because the logical state in the TTL technology is 5 volts for logic " 1 " as well as 0 volts for the "0". In the technology of FPGA the voltage of 5 volts by one of 3.3 volts replaced to achieve the " 1 " logic preserving 0volts as " 0 " the reading obtained by the accelerometer by a HC- 05 (transmitter) is sent, is received in an FPGA (Cyclone II) by another HC-05 (receiver), it is transmitted to the computer with RS-232 protocol and stored data on a computer for later analysis; (b) Sampling data optimal transmission curve in the devices of transmission data in FPGA. Also represents the voltage range of the system. 


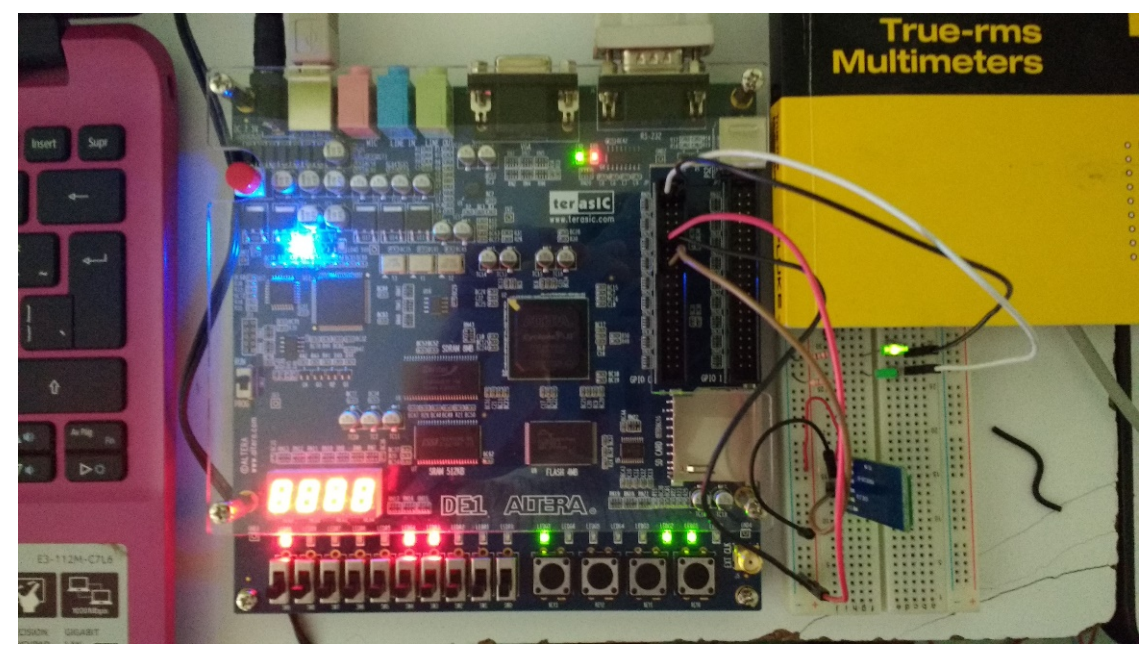

(a)

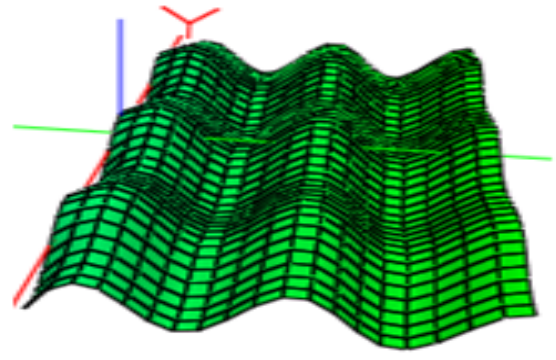

(b)

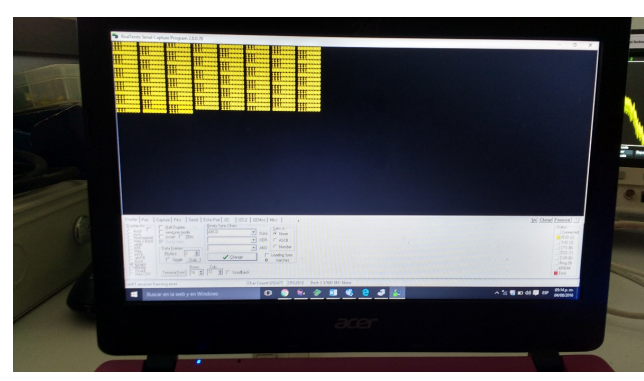

(c)

Figure 12. (a) Bluetooth module HC-05, which is found in the proto-board followed of the leds the Cyclone II, receiving the ASCII data and becoming these to binary codes in octaves. This decode can be observed in the red leds. The gate of the signals to the led is given for one of these two GPIO port of the Cyclone II. Finally, the arrangement Led to tests and proofs, given by the blue Led (see the block diagram given in the Figure 9(a), to understanding of this electronic module); (b) Surface of binary codes with variable electrical permittivity (axis $Y$ ) which has been modeled considering the permittivity given by $\varepsilon\left(t_{1}, t_{2}\right)=\left|\frac{D_{0}}{E_{0}}\right|\left(\cos \theta_{1}+\sin \theta_{2}\right)$. This define the data flown of the system. Also, this establishes an inter-relation between the $\omega$-space and the transmission space considering the properties of good RS-232 system. Observe the wave form to each period $T_{t}$, over the $Y$-axis; (c) Monitor of computer with octets of binary codes $(N=8)$.

The transmission optimization will be normed by the energy coefficients to the digitalized signal of $\kappa_{s}\left(\omega_{1}, \omega_{2}\right)$, whose energy consume is registered by the red Leds in the Cyclone II platform (Figure $12(\mathrm{a}))^{14}$ :

$$
\kappa_{l}=\frac{1}{l_{j} l_{i}} \int_{0}^{T_{0}} \int_{0}^{T_{0}} \kappa\left(t_{1}, t_{2}\right) \varphi_{l}^{*}\left(t_{1}, t_{2}\right) \mathrm{d} t_{1} \mathrm{~d} t_{2}, \quad l=1,2,3, \cdots, N
$$

which is the optimal way of data detection when the received signal could be corrupted for additive white noise. Here the coefficients (21) represent the digital data ready to be ${ }^{14}$ Here the functions $\varphi_{l}\left(t_{1}, t_{2}\right)$, with $l=1,2,3, \cdots, N$, also with $0<t_{1}<T_{t}^{1}$, and $0<t<T_{t}^{2}$, (we restrict to $T_{t}^{1}=T_{t}^{2}$ ) are orthogonal $N$ functions that give form to the wave. 
become them in binary codes [9] [10]. The space of this coefficient is $\mathcal{L}\left(H_{s}^{2}\left(\mathbb{Z}_{0,1}\right)\right)$, which appears in the storage and analyzed data in the monitor (Figure 12(c)). The binary codes surface is in the space $\mathcal{L}\left(H_{s}^{2}\left(\mathbb{Z}_{0,1} \times \Omega_{t}\right)\right)$.

\section{Conclusions}

The gravitational energy factor to design an accelerometer to a universal curvature sensor brings with it an "available" energy factor for the proper curvature in any curved space (included the Universe) bringing profit as "energy plus" to the proper mobile curvature sensor device. Likewise, due to the problem transmission optimization of curvature data we can enounce the following Table 1, of energy amplitudes used in this prototype II of curvature sensor, considering the complete system.

The future work of this research will design other devices of solid state that realize this profit of energy using the curvature energy obtained in downs and ups, or due to the positioning of the mobile device in the space (see the prospective to an advanced design of energy curvature sensor in the Universe to measure their curvature installed in an aerospace ship).

As it has been said, in the Section 4, and through the modulation analysis of pulse width PWM, the variations in raising or lowering show a relation of the signal with the inertia of the servo-system in their constant movement (see the Figure 9(a)) and thus also with the variation of the electric field inside the sensor device (our gauge field in this energy integrals theory) due to their respective variable capacitance which is consigned in position and thus is translated in curvature measure (see the Figure $9(\mathrm{c})$ )). The laboratory conditions comply with the band-width $\omega_{s} \leq 2 B \mathrm{~Hz} \approx 10 \mathrm{~Hz}$. The mobile curvature sensor device is limited in this frequency range due to the low constant velocity used for detection and monitoring of the curved space which is $\left\|\nabla_{X} Y\right\| \leq(\pi \times 0.06)\left[I(s)(R+L s) K_{1}\right]$.

We can conclude that the synchronization problem in the transmission process (movement of curvature sensor and the data transmission problem) stays normed by the lemma of regularity 2.1, which must establish as norm of the device displacement velocity such that this last velocity is constant.

Table 1. Energy amplitudes.

\begin{tabular}{cccc}
\hline \multirow{3}{*}{ Table Head } & \multicolumn{3}{c}{ Complete Energy System } \\
\cline { 2 - 4 } & Energy Amplitude & Wave Type & Band-width \\
\hline 1 & $C_{k}$ & $\kappa_{s}\left(\omega_{1}, \omega_{2}\right)$ & $\omega_{s} \leq 2 B \mathrm{~Hz}$ \\
2 & $\kappa_{l}$ & $\kappa\left(t_{1}, t_{2}\right)$ & $B \geq \frac{N}{2 T_{0}}=\frac{1}{2} D \mathrm{~Hz}$ \\
3 & $8 \pi$ & $\mathcal{\Theta}_{\mathrm{E}} / r^{2}$ & $\infty$ \\
4 & $D_{n}$ & $\kappa\left(\omega_{1}, \omega_{2}\right)$ & $\leq|H(\omega)|^{2} B$ \\
\hline
\end{tabular}


The next research that will be realized is the adjustment of constant movement of the mobile curvature sensor device and will be used to realize a terrestrial relief exploring to data of their curvature and obtain a curvature map. Finally the circuital optimizing of the sensor will be searched to increase their profit or yield in energy, and likewise decrease their loss of energy due to the heat or their transmission efficiency due to the electromagnetic scattering or signal dissipation.

\section{Acknowledgements}

We are very grateful with Edgar Daniel Sánchez Balderas, Law B, Principal of TESCHA, Evaristo Vázquez, Hernández, LC, B, Financial Sub-principal, and Rene Rivera Roldán, Electronic Eng., Electronic Division Chief, by their material support and facilities to realize this research.

\section{References}

[1] Bulnes, F., Martínez, I., Mendoza, A. and Landa, M. (2012) Design and Development of an Electronic Sensor to Detect and Measure Curvature of Spaces Using Curvature Energy. Journal of Sensor Technology, 2, 116-126. https://doi.org/10.4236/jst.2012.23017

[2] Bulnes, F., Martínez, I. and Zamudio, O. (2016) Fine Curvature Measurements through Curvature Energy and their Gauging and Sensoring in the Space. IFA Sensor Book.

[3] Bulnes, F., Martínez, I., Zamudio, O. and Negrete, G. (2015) Electronic Sensor Prototype to Detect and Measure Curvature through Their Curvature Energy. Science Journal of Circuits, Systems and Signal Processing, 4, 41-54. https://doi.org/10.11648/j.cssp.20150405.12

[4] Bulnes, F. (1998) Superior Mathematics Treatise: Signal and System Analysis, ETCFISMAT, UNAM, Science Faculty, Mexico.

[5] Bulnes, F. (2010) Research on Curvature of Homogeneous Spaces. TESCHA, Mexico, 4466. http://www.gimathematics.org

[6] Kobayashi, K. and Nomizu, K. (1969) Foundations of Differential Geometry. Wiley and Sons, New York.

[7] Sedra, A.S. and Smith, K.C. (2004). Microelectronic Circuits. 5th Edition, Oxford University Press, New York, 509.

[8] Zou, W.Y. (1991) Comparison of Proposed HDTV Terrestrial Broadcasting Systems. IEEE Transactions on Broadcasting, 37, 145-147. https://doi.org/10.1109/11.106202

[9] Oppenheim, V. and Schafer, R.W. (1989) Discrete-Time Signal Process. Prentice Hall, Upper Saddle River.

[10] Benedetto, S., Biglieri, E. and Castellani, V. (1987) Digital Transmission Theory. Prentice Hall, Upper Saddle River. 


\section{Technical Notation and Abbreviations}

$K$-Curvature as general concept of roundness property. Also used in the paper as Gaussian curvature in a point $p$.

$k-$ Gaussian curvature along of the geodesic or surface.

-Sensor device as black box. This simplified representation of our electronic sensor is useful to show the goals of curvature exploring of surfaces.

$\mathcal{O}_{E}$-Spherical operator which is related with the spherical map. Also is the energy of a sphere of radius $r$.

USB-SERIAL-Storage-connection cable to binary signal recollecting.

$V$-Voltage.

FPGA-Field Programmable Gate Array. This is a programmable device that include logic blocks whose interconnection and functionality can be configured "in situs" through a language of specialized description.

ASCII-American Standard Code for Information Interchange. Also named usually as "aski code", which is a character code based in the Latin alphabet, such as is used in modern English

$\nabla_{X} Y$-Shape operator. This is an operator to measure the velocity of change of direction of one field respect to other field. In our sensor the gauge field (electrical field) varies respect to reference systems field (position average field).

$\operatorname{Hom}_{K}(M, \Sigma)$-Spherical mappings space, which establish the mapping of the surface used to measure their curvature $M$, and the sphere $\Sigma$, of certain radius $r$.

A-Area.

$\kappa\left(\omega_{1}, \omega_{2}\right)$-Spectral curvature in the Fourier space.

TTL-Technology model based in "logic from transistor to transistor", (transistor-transistor logic).

PCL_Programmable Logic Controller.

Bluetooth HC-05 Module-It's data transmission system of master-slave module, which has advantages in intercommunication in a closed electronic system.

GPIO-General Purpose Input/Output. It's a generic pin in a chip, whose behavior (including, if is an input or output pin) can be controlled (programmed) by the user in time of execution.

Cyclone II-Electronic platform or board with low cost and optimized feature set of FPGAs make them ideal solutions for a wide array of automotive, consumer, communications, video processing, test and measurement, and other end-market solutions. Reference designs, system diagrams.

RS-232-Recommended Standard 232. Also known as EIA/TIA RS-232C, is an interphase that designs a norm to the interchange of a binary data series between a DTE (Data Terminal Equipment) and a DCE (Data Communication Equipment), although exist other in the that also is used the interphase RS-232. Also is known as V.24.

$\kappa_{s}\left(\omega_{1}, \omega_{2}\right)$-Sampling spectral curvature in the $\omega$-space.

$\mathcal{L}\left(H_{s}^{2}\left(\mathbb{Z}_{0,1}\right)\right)$-Binary Spectral Curvature Space. 
$\kappa\left(t_{1}, t_{2}\right)$ - Real spectral curvature. This is the measured by electrical devices directly of the surfaces.

$\mathrm{B}_{\mathrm{E}}^{\mathrm{C}}$-Bipolar Transistor. A bipolar junction transistor (bipolar transistor or BJT) is a type of transistor that uses both electron and hole charge carriers.

Submit or recommend next manuscript to SCIRP and we will provide best service for you:

Accepting pre-submission inquiries through Email, Facebook, LinkedIn, Twitter, etc.

A wide selection of journals (inclusive of 9 subjects, more than 200 journals)

Providing 24-hour high-quality service

User-friendly online submission system

Fair and swift peer-review system

Efficient typesetting and proofreading procedure

Display of the result of downloads and visits, as well as the number of cited articles

Maximum dissemination of your research work

Submit your manuscript at: http://papersubmission.scirp.org/

Or contact jst@scirp.org 